Discrete Comput Geom 31:323-326 (2004)

DOI: $10.1007 / \mathrm{s} 00454-003-2903-\mathrm{z}$

\title{
Aperiodic Linearly Repetitive Delone Sets Are Densely Repetitive
}

\author{
Daniel Lenz \\ Fakultät für Mathematik, TU Chemnitz, \\ D-09107 Chemnitz, Germany \\ dlenz@mathematik.tu-chemnitz.de
}

\begin{abstract}
We show that aperiodic linearly repetitive Delone sets are densely repetitive. This confirms a conjecture of Lagarias and Pleasants.
\end{abstract}

\section{Introduction}

In [2] Lagarias and Pleasants study the problem of characterizing the simplest aperiodic discrete point sets. To this end they carry out a careful study of linearly repetitive and densely repetitive Delone sets. As for the relationship between these two concepts they formulate the following conjecture:

Conjecture (= Conjecture 1.2a in [2]). Every aperiodic linearly repetitive Delone set is densely repetitive.

It is the aim of this note to prove the conjecture. The proof is a direct consequence of a reformulation of the conjecture in terms of lower bounds on local complexity as given already in [2] and two essentially known facts. The first fact is a certain repulsion property of occurrences of the same patches in aperiodic linearly repetitive Delone sets (see the work of Solomyak [5] and Durand [1]). The second is a connection between the absence of "local periods" and lower bounds on local complexity as studied, e.g., by the author in [4].

\section{Proof of the Conjecture}

We begin by recalling some necessary notation (mostly taken from [2]). A set $X \subset \mathbb{R}^{d}$ is called a Delone set if there exist $0<r(X), R(X)<\infty$ such that every ball in $\mathbb{R}^{d}$ 
with radius $r(X)$ meets at most one point of $X$ and every ball with radius $R(X)$ meets at least one point of $X$. For $T>0$, a set of the form $X \cap B(x, T)$ with $x \in X$ is called a $T$-patch centered at $x$. Here, $B(p, s)$ denotes the closed ball around $p$ with radius $s$. The number $N_{X}(T)$ of different $T$-patches up to translation is defined by

$$
N_{X}(T) \equiv \sharp\{(X-x) \cap B(0, T): x \in X\},
$$

where $\sharp S$ denotes the number of elements of the set $S$. The Delone set $X$ is called repetitive if, for every $T>0$, there exists a finite number $M$ such that every closed ball of radius $M$ in $\mathbb{R}^{d}$ contains the center of a translate of every possible $T$-patch in $X$. The smallest such $M$ is denoted by $M_{X}(T)$.

A Delone set $X$ is called linearly repetitive if there exists a constant $C_{\mathrm{LR}}(X) \in \mathbb{R}$ with $M_{X}(T) \leq C_{\mathrm{LR}}(X) T$ for every $T \geq 1$. It is called densely repetitive if there exists a constant $C_{\mathrm{DR}}(X) \in \mathbb{R}$ with $M_{X}(T) \leq C_{\mathrm{DR}}(X) N_{X}^{1 / d}(T)$ for $T \geq 1$. The set $X$ is called non-periodic if it is not equal to a translate of itself and it is called aperiodic if this property holds for all elements in the hull. Here, the hull is the closure of its translates in the natural topology [2]. For repetitive Delone sets non-periodicity and aperiodicity are equivalent.

Our proof of the conjecture is based on the following two lemmas. The first gives a precise version of the repulsion property mentioned in the Introduction.

Lemma 2.1. Let $X$ be an aperiodic linearly repetitive Delone set. Then there exists a constant $\kappa(X)>0$ such that $\|x-y\| \geq \kappa(X) T$ whenever $x, y \in X$ satisfy $x \neq y$ and $(X-x) \cap B(0, T)=(X-y) \cap B(0, T)$ for some $T>1$. Here $\|\cdot\|$ denotes the Euclidean norm.

For linearly repetitive tilings this lemma (and in fact a slightly stronger version) is proven in Lemma 2.4 of [5] (see the proof of Theorem 2.2 in [3] and [1] as well). The proof given there is formulated in terms of tilings associated to primitive substitutions but uses only linear repetitivity (called strong repetitivity in [5]). It can easily be carried over to Delone sets. For completeness reasons, we include a short discussion giving a

Proof of Lemma 2.1. Assume the contrary. Then there exist $x, y \in X, x \neq y$ with

$$
(X-x) \cap B(0, T)=(X-y) \cap B(0, T)
$$

and

$$
\|x-y\| \leq \frac{T}{\left(C_{\mathrm{LR}}+1\right)} .
$$

In particular, for every point $s \in X \cap B(x, T)$, the point $s+(y-x)$ belongs to $X$ as well. By linear repetitivity, $X \cap B(x, T)$ contains the center of a translate of every $C_{\mathrm{LR}}^{-1} T$-patch of $X$. As $C_{\mathrm{LR}}^{-1} T>\|x-y\|$, we infer that for every $z \in X$ the point $z+(y-x)$ belongs to $X$ as well. Hence $y-x$ is a period of $X$. This contradiction proves Lemma 2.1. 
The second lemma is a consequence of the first lemma.

Lemma 2.2. Let $X$ be an aperiodic linearly repetitive Delone set. Then there exist constants $\lambda>0$ and $T_{0}>0$ with $N_{X}(T) \geq \lambda T^{d}$ for $T \geq T_{0}$.

Proof. As $X$ is a Delone set, there exists $\lambda_{1}>0$ and $T_{1}>0$ with

$$
\sharp X \cap B(p, T) \geq \lambda_{1} T^{d}
$$

for all $p \in R^{d}$ and $T \geq T_{1}$. Now, let $\kappa(X)$ be as given in the previous lemma and consider for $T>1$ the $T$-patches

$$
(X-x) \cap B(0, T) \quad \text { with } \quad x \in X \cap B(0, \kappa(X) T / 3) .
$$

Then, by the previous lemma, these $T$-patches are pairwise different. Thus, using (1), we can calculate

$$
N_{X}(T) \geq \sharp(X \cap B(0, \kappa(X) T / 3)) \geq \lambda_{1}(\kappa(X) T / 3)^{d}=\lambda T^{d},
$$

for $T \geq T_{0} \equiv 3 \kappa(X)^{-1} T_{1}$ and $\lambda=\lambda_{1}(\kappa(X) / 3)^{d}$.

We can now provide the

Proof of the Conjecture. It suffices to show

$$
\liminf _{T \rightarrow \infty} \frac{N_{X}(T)}{T^{d}}>0
$$

In fact, by the discussion in Section 8 of [2] (see [3] as well), the validity of (2) is even equivalent to the validity of the conjecture. Now, (2) follows immediately from the previous lemma. The conjecture is proven.

Remark 1. It is not hard to see that the aperiodicity assumption in the conjecture is essential: For example, the product of a fully periodic set with a linearly repetitive set is linearly repetetive but not densely repetitive.

\section{Acknowledgments}

The author thanks Boris Solomyak for stimulating discussions. He also thanks Peter Pleasants for various useful comments yielding, in particular, Remark 1.

\section{References}

1. F. Durand, Linearly recurrent subshifts have a finite number of non-periodic subshift factors, Ergodic Theory Dynamical Systems 20 (2000), 1061-1078.

2. J. C. Lagarias and P. A. B. Pleasants, Repetitive Delone sets and quasicrystals, Ergodic Theory Dynamical Systems, 23 (2003), 831-867. 
3. J. C. Lagarias and P. A. B. Pleasants, Local complexity of Delone sets and crystallinity, Canad. Math. Bull. 45 (2002), 634-652.

4. D. Lenz, Hierarchical structures in Sturmian dynamical systems, Theoret. Comput. Sci. 303 (2003), 463-490.

5. B. Solomyak, Nonperiodicity implies unique composition for self-similar translationally finite tilings, Discrete Comput. Geom. 20 (1998), 265-279.

Received August 18, 2002, and in revised form October 18, 2002. Online publication December 19, 2003. 